\title{
Efecto antagonista del flumazenil sobre el isoflurano en la emersión de la anestesia general
}

\section{Antagonist effect of flumazenil on isofluran in reversion of general anesthesia}

Javier Eduardo Moreno Sánchez' ${ }^{1}$ J Jonathan De Freitas H. ${ }^{1}$, Dra. María Victoria Amaro S. ${ }^{1}$

\begin{abstract}
Introduction and Objectives: Isoflurane, an inhalational general anesthetic widely used in medical practice, belonging to the group of volatile liquids together with desflurane and sevoflurane, with various properties including sedation, hypnosis and anesthesia of patients undergoing treatment. surgical acts. Volatile inhalational anesthetics (halogenated) as mechanism of action, has the property of increasing inhibitory synaptic transmission at postsynaptic level by potentiating ion channels regulated by ligand activated by alpha-aminobutyric acid (GABA). Flumazenil is a benzodiazepine antagonist belonging to the group of imidazobenzodiazepine. It is currently known that there is no specific drug capable of antagonizing the effects of halogenates that allow the rapid and complete recovery of general anesthesia, for this reason this work focuses its efforts on demonstrating whether flumazenil has the ability to reverse the actions of the patient. isoflurane and allow an early restoration of the level of consciousness. Materials and Methods: The study to be performed is a clinical type of longitudinal, prospective, unicentric and double blind. The sample will be formed by patients who are going to be subjected to a balanced general anesthesia. The sample will be divided into 2 large groups: group C (control) and group $F$ (Flumazenil). At the end of the surgery, the mixture will be administered according to the selected group in a random manner (Flumazenil 0.25 $\mathrm{mg}$ or $0.9 \%$ solution in a $20 \mathrm{cc}$ syringe) and the time of extubation, recovery time of the level of consciousness, time of discharge UCPA and hemodynamic state (FC, TAM and $\mathrm{SO}_{2}$ ). Results: The flumazenil group showed a significantly shorter time from injection to extubation than the placebo group $(p=0.007)$. Differences in terms of shorter times needed to achieve Aldrete of 9 points in
\end{abstract}

Key words:

Flumazenil, isoflurane, antagonism, extubation, receptors, GABA, anesthesia

Servicio de Anestesiología y Reanimación, Hospital Central de Maracay. Edo. Aragua, Venezuela.

Fecha de ingreso: 24 de junio de 2019

Fecha de aceptación: 30 de julio de 2019

\section{ORCID}

https://orcid.org/0000-0002-9785-0735

Correspondencia:

Dr. Javier Eduardo Moreno Sánchez

drjavieremorenos@hotmail.com 
the flumazenil group $(P=0.04)$ were observed as were shorter anesthetic arousal times represented by a Ramsey 2 . Heart rate, mean arterial pressure and saturation they had similar values between the 2 groups. Conclusion: The study showed that a single dose of $0.25 \mathrm{mg}$ of flumazenil administered at the end of the surgical act, just after completing all surgical stimulation was beneficial $(P=$ $0.007)$ in the context of extubation times and shorter anesthetic arousal times.

\section{RESUMEN}

Introducción y Objetivos: El isoflurano un anestésico general inhalatorio usado ampliamente en la práctica médica, perteneciente al grupo de los líquidos volátiles junto con el desflurano y sevoflurano, con variadas propiedades entre las que se encuentran la sedación, hipnosis y anestesia de los pacientes sometidos a actos quirúrgicos. Los anestésicos inhalatorios volátiles (halogenados) como mecanismo de acción, tiene la propiedad de aumentar la transmisión sináptica inhibidora a nivel postsináptico potenciando los canales iónicos regulados por ligando activados por ácido alfa-aminobutírico (GABA). El flumazenil es un antagonista benzodiazepínico perteneciente al grupo de los imidazobenzodiazepina. Se conoce actualmente que no existe un fármaco específico capaz de antagonizar los efectos de los halogenados que permitan la recuperación rápida y completa de la anestesia general, por tal motivo este trabajo centra sus esfuerzos en demostrar si el flumazenil tiene la capacidad para revertir las acciones del isoflurane y permitir un restablecimiento temprano del nivel de conciencia. Materiales y Métodos: El estudio a realizar es de tipo clínico de corte longitudinal, prospectivo, unicéntrico y doble ciego. La muestra se conformará por pacientes que vayan a ser sometidos a anestesia general balanceada. Se procederá a dividir la muestra en 2 grandes grupos: grupo C (control) y grupo $\mathrm{F}$ (flumazenil). Al final de la cirugía se administrará la mezcla según grupo seleccionado de manera al azar (flumazenil 0,25 mg o solución 0,9\% en una jeringa de 20 cc) y se valorará el tiempo de extubación, tiempo de recuperación del nivel de conciencia, tiempo de alta de la UCPA y estado hemodinámico ( $\mathrm{FC}, \mathrm{TAM}$ y $\mathrm{SO}_{2}$ ). Resultados: El grupo de flumazenil presentó un tiempo desde la inyección hasta la extubación significativamente más bajo que el grupo placebo $(p=0,007)$. Se observaron diferencias en términos de tiempos más bajos necesario para alcanzar Aldrete de 9 puntos en el grupo flumazenil $(P=0,04)$ al igual que tiempos de despertar anestésico más cortos representados por un Ramsey 2. La frecuencia cardíaca, presión arterial media y la saturación tuvieron valores similares entre los 2 grupos. Conclusión: El estudio demostró que una única dosis de $0,25 \mathrm{mg}$ de flumazenil administrado al final del acto quirúrgico, justo después de culminar toda estimulación quirúrgica fue beneficiosa $(P=0,007)$ en el contexto de tiempos de extubación y tiempos de despertar anestésico más cortos.

\section{Palabras clave:}

Flumazenil, isoflurano, antagonismo, extubación, receptor, GABA, anestesia

\section{Introducción}

E I isoflurano es un anestésico general inhalatorio usado ampliamente en la práctica médica, perteneciente al grupo de los líquidos volátiles junto con el desflurano y sevoflurano, con variadas propie- dades entre las que se encuentran la sedación, hipnosis y anestesia de los pacientes sometidos a actos quirúrgicos[1].

Los anestésicos inhalatorios volátiles (halogenados) como mecanismo de acción, tiene la propiedad de aumentar la transmisión sináptica inhibidora a 
nivel postsináptico potenciando los canales iónicos regulados por ligando activados por ácido alfaaminobutírico (GABA) y glicina, a nivel extrasináptico potenciando los receptores GABA y las corrientes de fuga, y a nivel presináptico aumentando la liberación basal de GABA. De manera clásica los anestésicos inhalatorios suprimen la transmisión sináptica excitatoria a nivel presináptico reduciendo la liberación de glutamato, y a nivel postsináptico inhibiendo los receptores ionotrópicos excitadores activados por glutamato[1],[2].

El flumazenil es un antagonista benzodiazepínico perteneciente al grupo de los imidazobenzodiazepina. Se trata de un agonista parcial con una mínima actividad intrínseca y muy alta actividad antagonista, de forma que a dosis muy elevadas se pone de manifiesto cierta acción anticonvulsivante, mientras que en otros estudios muestra una pequeña acción de tipo agonista inverso[3],[6].

El fármaco se liga muy ávidamente a sitios específicos del receptor GABA A, en los cuales muestra antagonismo competitivo con la unión y los efectos alostéricos de las benzodiazepinas y otros ligandos. Se antagonizan los efectos tanto electrofisiológicos como del comportamiento del agonista o de las benzodiazepinas agonistas. En estudios en animales, las acciones farmacológicas intrínsecas del flumazenil han sido leves por lo que sus acciones se concentran en antagonizar los efectos por las benzodiazepinas[3],[4],[8].

Su principal utilidad terapéutica radica en la práctica anestésica, para revertir la sedación, hipnosis y anestesia provocada por benzodiazepinas. Así mismo puede ser de utilidad para el diagnóstico diferencial de la intoxicación benzodiazepínica y para su tratamiento[5],[7].

Se sabe que la anestesia no es una técnica completamente exenta de riesgos y que la extubación y recuperación temprana de la misma, así como un alta precoz de la Unidad de Recuperación Postanestésica disminuye la incidencia de complicaciones y morbilidad en los pacientes. Se conoce, actualmente, que no existe un fármaco específico capaz de antagonizar los efectos de los halogenados que permitan la recuperación rápida y completa de la anestesia general, por tal motivo este trabajo centra sus esfuerzos en demostrar a través de parámetros hemodinámicos y clínicos si el flumazenil tiene la capacidad para revertir las acciones del isoflurane y permitir un restablecimiento temprano del nivel de conciencia, una extubación temprana, menor índice de complicaciones portoperatorias y una alta precoz de la Unidad de Cuidados Postanestesica[2],[5].

Múltiples ensayos clínicos intentan poner en ma- nifiesto la capacidad que tiene el flumazenilo para revertir la sedación, hipnosis y anestesia provocada por los anestésicos inhalatorios; específicamente el isoflurane, dirigiendo sus hipótesis a las acciones que el halogenado ejerce sobre sitios específicos del receptor GABA A, actuando como potenciador de la inhibición del GABA, efecto que puede ser antagonizado teóricamente por el flumazenilo. Este receptor, descrito hace un tiempo en la fisiología del cerebro, es el sitio de unión de varios anestésicos, como las benzodiazepinas y los barbitúricos[6].

Se ha demostrado que los fármacos anestésicos inhalatorios se relacionan muy estrechamente con un despertar anestésico lento y bastante desagradable para el paciente caracterizado principalmente por letargia, agitación psicomotriz y desorientación; así como un mayor índice de depresión respiratoria que puede coincidir con la presencia de broncoaspiración y en los peores casos reintubación o muerte. Por lo anterior descrito el presente trabajo justifica sus estudios en evitar la aparición de dichas complicaciones al demostrar la capacidad que tiene el flumazenilo de revertir los efectos del isoflurano[3],[5].

En la actualidad se ha visto con mucha preocupación cómo ha aumentado el número de pacientes que acuden cada año a los centros hospitalarios buscando ayuda. Centros hospitalarios que se encuentran ocupados en más de $80 \%$ y aun así miles de visitas al mes de pacientes que ameritan hospitalización por diversas causas. Una de ellas es la necesidad de resolución de una patología médica quirúrgica. Patología que mecere de cuidados preoperatorios y postoperatorios que generan grandes gastos al centro hospitalario y en última instancia al Estado. Con el uso del flumazenilo se pretende demostrar su utilidad en la emersión de la anestesia general con isoflurane que conlleve a una recuperación más rápida, el alta precoz de UCPA lo que originarán una disminución del tiempo de estadía y menores costos hospitalarios.

\section{Materiales y Métodos}

\section{Tipo de estudio}

El estudio a realizar es de tipo clínico de corte longitudinal, prospectivo, unicéntrico y doble ciego en el que se comparará la eficacia del flumazenilo para revertir los efectos hipnóticos de los halogenados, en este caso, el agente seleccionado es el isoflurano en pacientes sometidos a anestesia general balanceada en el Servicio Autónomo Hospital Central de Maracay desde abril - octubre de 2017. 


\section{Población y Muestra}

La población estará conformada por pacientes que acudan al área quirúrgica del Servicio Autónomo Hospital Central de Maracay para ser intervenidos quirúrgicamente, la muestra se conformará por pacientes que vayan a ser sometidos a anestesia general balanceada en el periodo comprendido entre abril octubre de 2017 en el Servicio Autónomo Hospital Central de Maracay y que cumplan con los criterios de inclusión y exclusión mencionados a continuación:

\section{Criterios de inclusión}

- Pacientes adultos de ambos sexos.

- Edades comprendidas entre 18 y 65 años.

- Buen estado de salud o enfermedades leves completamente controladas por el uso regular de medicamentos (ASA I o II, según el esquema de clasificación de la American Society of Anesthesiologists).

\section{Criterios de exclusión}

- Historia o presencia de enfermedades neurológicas, convulsiones o trastorno de pánico.

- Historia de hipertermia maligna.

- Trastornos de la audición.

- Uso crónico o premedicación con benzodiazepinas.

- Uso crónico de drogas estupefacientes.

- Paciente con patología agudo o crónica hepática o renal.

- Pacientes con patologías pulmonares agudas o crónicas.

- Pacientes en shock o hemodinámicamente inestable.

\section{Procedimiento clínico}

Los autores se dirigirán al área quirúrgica del Servicio Autónomo Hospital Central de Maracay en donde se seleccionará a los pacientes que se encuentran en la estación preanestésica que vayan a ser sometidos a anestesia general balanceada, se procederá a seleccionar de estos pacientes, solo aquellos que cumplan con los criterios de inclusión y que no tengan criterios de exclusión, se explicará al paciente en qué consiste el estudio y se procederá a realizar la firma del consentimiento informado. Posterior a esto, se procederá a dividir la muestra en 2 grandes grupos: grupo C (control) y grupo F (flumazenil). Esta división se realizará de manera aleatoria estandarizada, me- diante técnica de sobre cerrado: en un grupo C conformado por los pacientes que recibirán el placebo y el grupo $F$ aquellos a los que se les administrará el bolo de flumazenil una vez se suspenda la administración del mantenimiento inhalatorio con isofluorano.

Una vez seleccionado el grupo de estudio, se procederá a pasar a quirófano en donde se realizará la intervención quirúrgica, es importante que el mismo no debe ser premedicado con ningún tipo de benzodiazepinas ni opioides, se monitorizará al paciente y se procederá a realizar la inducción anestésica: se preoxigenará al mismo con oxígeno $100 \%$ a razón de 6 I-min, la inducción se realizará de manera endovenosa con fentanilo $3 \mathrm{mcg} / \mathrm{kg} /$ dosis + lidocaína $1 \mathrm{mg} /$ $\mathrm{kg} /$ dosis + propofol $2,5 \mathrm{mg} / \mathrm{kg} /$ dosis y rocuronio 0,6 $\mathrm{mg} / \mathrm{kg} / \mathrm{dosis}$; se realizará intubación orotraqueal y se conectará a sistema cerrado de recirculación parcial con canister, se someterá a ventilación mecánica; el mantenimiento anestésico se realizará por vía inhalatoria con isoflurane a razón de $1,15 \mathrm{Vol} \%$ con un flujo de 2 litros de oxígeno al 100\%; y endovenosa con fentanilo $1 \mathrm{mcg} / \mathrm{kg} / \mathrm{dosis}$ y rocuronio $10 \mathrm{mg}$ cada 35 min. Como coadyuvantes de la anestesia se administrarán en el intraoperatorio a cada paciente protector gástrico (ranitidina $50 \mathrm{mg}$ ), antieméticos (ondasetron $4 \mathrm{mg}$ ) y analgésicos (dipirona $2 \mathrm{~g}$ ), mientras no estén contraindicados.

Al final del acto quirúrgico, todos los pacientes recibirán de manera intravenosa una infusión de dos soluciones preparadas previamente, según el grupo al cual hayan sido asignados al azar. El grupo $C$ recibirá $20 \mathrm{~mL}$ de solución salina y el grupo $\mathrm{F}$ recibirá $0,25 \mathrm{mg}$ de flumazenil (diluidos en $20 \mathrm{~mL}$ de solución salina). Ambas soluciones se administrarán en una infusión intravenosa, inmediatamente después de haber culminado la administración de isoflurano.

El anestesiólogo no tendrá conocimiento del contenido de la solución a infundir y procederá a evaluar: 1) el plano anestésico del paciente al minuto (T1) durante los primeros 5 minutos (T5), luego a los 10 minutos (T10) y a los 15 minutos (T15); 2) calcular el tiempo que transcurra desde la suspensión del isoflurano hasta que el paciente recobra totalmente consciencia evidenciando una escala de ramsay 2 y en donde es capaz de mantener una conversación coherente con el operador; 3) calcular el tiempo que transcurra desde la suspensión del isoflurano hasta momento de la extubacion; 4) monitorizar los signos vitales durante el proceso de extubacion al $1^{\text {er minuto }}$ (T1), a los 5 min (T5), a los 10 (T10) y a los $15 \mathrm{~min}$ (15) posteriores al cierre del isoflurano; 5) registrar la presencia de alguna complicación o reacción adversa en caso de evidenciarse y 6) determinar el tiempo de 
estadía en la Unidad de Cuidados Postanestésicos.

El estudio fue aprobado por el Comité de Bioética del Hospital Central de Maracay. El estudio se llevó a cabo en los quirófanos del Hospital Central de Maracay en el periodo abril - octubre de 2017. Después de recibir la invitación de participar en el estudio y la información sobre el mismo, todos los participantes firmaron un documento de consentimiento informado.

\section{Resultados}

De los 66 pacientes que fueron incluidos inicialmente en el estudio, se excluyeron 2 debido a un retardo en el despertar anestésico el cual no se corresponde con los tiempos habituales del anestésico general inhalatorio usado en el estudio.

Los otros 64 pacientes siguieron en el estudio hasta el final (alta de la sala de recuperación postanestésica) y sus datos fueron analizados. No hubo diferencias significativas entre los 2 grupos en términos de sexo y edad (Tabla 1), pero sí se encontró diferencia en cuanto al tiempo quirúrgico, siendo representado por el 40,6\% aquellas cirugías con una duración de $90 \mathrm{~min}$.

El grupo de flumazenil presentó un tiempo desde la inyección hasta la extubación significativamente más bajo que el grupo placebo $(p=0,007)$. Se observaron diferencias en términos de tiempos más bajos necesario para alcanzar Aldrete de 9 puntos en el grupo flumazenil $(P=0,04)$ al igual que tiempos de despertar anestésico más cortos representados por un Ramsey 2 (Tabla 2). La frecuencia cardíaca, presión ar- terial media y la saturación tuvieron valores similares entre los 2 grupos (Figura 1).

En todo el estudio ningún paciente mostro complicaciones ni reacciones adversas al flumazenil (convulsiones, agitación, ansiedad).

Las variables obtenidas de las muestras fueron agrupadas y almacenadas en una hoja de cálculos de Excel 97/2003 como base de datos. Como programas estadísticos se usó Epilnfo versión 3.5.1 para la correlación de los resultados a través de diferentes estudios estadísticos usando las frecuencias y medias de cada variable. Se usó el programa JMP 5.1 para correlacionar los datos de las variables continuas.

\section{Discusión}

El flumazenil tiene un gran poder antagonista indicado para neutralizar las excesivas medicaciones

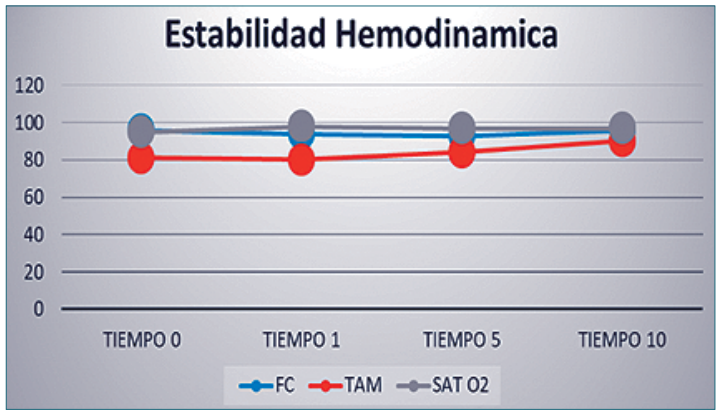

Figura 1. Cambios hemodinámicos.

\begin{tabular}{lccc}
\multicolumn{4}{c}{ Tabla 1. Características demográficas de la muestra } \\
\hline Demográficos & Control & Flumazenil & Total \\
Sexo (M/F) & $12 / 19$ & $18 / 15$ & $30 / 34$ \\
Edad & 39 & 40 & 40 \\
\hline
\end{tabular}

\begin{tabular}{lccc}
\multicolumn{2}{c}{ Tabla 2. Comparación de los grupos en términos de los parámetros estudiados } \\
\hline Parámetro & Control & Flumazenil & P \\
TAM (mmhg) & $84+-3$ & $82+-5$ & NS \\
FC (lpm) & $94+-1$ & $95+-2$ & NS \\
Saturación $\mathrm{O}_{2}(\%)$ & $96+-2$ & $97+-1$ & NS \\
Tiempo extubación* & 10,96 & 7,46 & 0,007 \\
Tiempo Ramsey* & 22,18 & 20,68 & NS \\
Tiempo Aldrete* & 34,66 & 28,61 & 0,04 \\
\hline
\end{tabular}


con benzodiazepinas o en la recuperación de la sedación/hipnosis tras una cirugía, esto a través de la inhibición de los receptores de GABA en el sistema nervioso central. Este efecto del flumazenil es causado específicamente por inhibición competitiva de los receptores GABA, los objetivos de las benzodiazepinas. Receptor que juega un papel importante sobre el mecanismo de acción de los anestésicos generales inhalatorios específicamente el isoflurano y su relación estrecha con el GABA[3],[5].

En este estudio que se llevó a cabo en pacientes no premedicados y que no recibieron benzodiazepinas demostró que la inyección de 0,25 mg de flumazenil al final del acto quirúrgico, justo después de culminar la estimulación quirúrgica redujo significativamente ( $p=0,007$ ) el tiempo necesario para la extubación y recuperación de la anestesia general con isoflurano en comparación con el grupo control, así como tiempos más cortos para obtener un Aldrete de 9 puntos o más $(P=0,04)$ en la UCPA. Sin embargo, existen múltiples estudios que ponen en duda dicha relación entre las benzodiazepinas y el isoflurano, debido al mecanismo de acción poco claro de los anestésicos inhalatorios[10],[11]. Schwieger informó que el flumazenil no afecta a la MAC de enflurano, isoflurano, o una combinación de fentanilo-enflurano[13]; pero en este estudio se pudo demostrar la efectividad del flumazenil para revertir o antagonizar los efectos del isoflurano y que corresponderían a tiempos de despertar más cortos.

Domingos Días Cicarelli en el 2016, concluyó en su estudio que una dosis de $1 \mathrm{mg}$ de flumazenil al final de la cirugía fue efectivo en reducir el tiempo de extubación de pacientes sometidos a anestesia general por isoflurano[12]. Así mismo, Yi Jeong Kim en el
2012, llevó a cabo un estudio en pacientes que no recibieron benzodiazepinas y los resultados mostraron que la inyección de 0,3 mg de flumazenil al final de la anestesia redujo significativamente el tiempo necesario para la recuperación de la anestesia y aumentó el valor de BIS en comparación con el grupo control[14].

Recientemente, Karakosta, descubrió que 0,3 mg de flumazenil puede mejorar los parámetros de recuperación de la anestesia con sevoflurano/remifentanilo después de la administración a pacientes no premedicados 30 minutos antes del final de la operación[15]. A diferencia de los estudios anteriores pudimos demostrar que con una sola dosis de 0,25 mg de flumazenil es suficiente para antagonizar los efectos del isoflurano y que no son necesarias dosis más altas como 0,5 mg o $1 \mathrm{mg}$ previamente descritas en la bibliografía.

\section{Conclusión}

El estudio demostró que una única dosis de 0,25 mg de flumazenil administrado al final del acto quirúrgico, justo después de culminar toda estimulación quirúrgica, fue beneficiosa ( $P=0,007)$ en el contexto de tiempos de extubación y tiempos de despertar anestésico más cortos en comparación con aquellos que no lo recibieron. Los pacientes de este estudio también se vieron beneficiados por presentar periodos de estadía más cortos en la UCPA $(P=0,04)$ valorados por un Aldrete mayor a 9 puntos en aquellos que recibieron flumazenial a final del acto quirúrgico y cambios hemodinámicos mínimos en ambos grupos.

\section{Referencias}

1. Ronald d. Miller, md. Miller anestesia. Séptima edición. Editorial Elsevier España sl. Año 2010. volumen 1, sección II, capítulo 10 anestésicos inhalatorios: mecanismos de acción, pag 281304.

2. Jesús Flores. Farmacología humana. Quinta (5ta) edición. Año 2008. Editorial Elsevier Masson. Sección IV sistema nervioso central, capitulo 28 fármacos anestésicos generales, pag 567-
578.

3. Laurence I. Brunton, phd. Goodman \& Gilman las bases farmacológicas de la terapéutica. Undecima segunda edición. Año 2007. Editorial Mc Graw Hill. Sección III fármacos con acción en el sistema nervioso central, apítulo 16 hipnóticos y sedantes, pag 401-428.

4. J. Antonio Aldrete, Uriah Guevara Lopez y Emilio M. Capmourteres. Texto de Anestesiologia Teorico-practico. Segunda edición. Editorial El Manual Moder- no. Año 2004. Seccion III, capitulo 13 inductores anestésicos, pag $225-242$.

5. Barash, Paul G.; Cullen, Bruce F.; Stoelting, Robert K. Clinical anesthesia. 5ta edicion. Editorial Lippincott Williams \& Wilkins. Año 2006. Seccion III basic principles of clinical pharmacology, chapter 13 nonopioid intravenous anesthesia, pag 687 - 727.

6. Mcmillan co, spahr-schopfer ia, sikich $n$, hartley e, lerman j (1992) premedication of children with oral midazolam. Can j 
anaesth 39:545-550. https://doi. org/10.1007/BF03008315

7. Amrein $R$, Hetzel $W$, Hartmann $D$, Lorscheid T. Clinical pharmacology of flumazenil. Eur $\mathrm{j}$ anaesthesiol suppl. 1988;2:6580. [pubmed].

8. Guyton y hall. Tratado de fisiología médica 11 edicion en español. Elsevier saunders. 2006. Pag 175-176.2.

9. Anthony s. Fauci, eugene braunwald. Harrison principios de medicina interna 17 edicionen español. Mc graw hill. 2009. Capítulo 261 pag 1673.

10. Peng liang, cheng zhou, kai-yu li, li-juan guo, bin liu, jin liu. Effect of flumazenil on sevoflurane requirements for minimum alveolar anesthetic concentrationawake and recovery status. Department of anesthesiology, west china hospital, sichuan university, chengdu, sichuan, china, jinzhong, shanxi, china. Received october 21, 2013; accepted november 5, 2013; epub march 15, 2014; published march 30, 2014. Int j clin exp med 014;7(3):673-679

11. Seyed a. Safavynia, m.d., ph.d., glenda keating, ph.d., iris speigel, b.s., jonathan a. Fidler, b.s., matthias kreuzer, ph.d., david b. Rye, m.d., ph.d., andrew jenkins, ph.d., paul s. García, m.d., ph.d. effects of $\gamma$-aminobutyric acid type a receptor modulation by flumazenil on emergence from general anesthesia. The american society of anesthesiologists. Anesthesiology, v $125 \bullet$ no 1 . Julio 2016. https://doi.org/10.1097/ ALN.0000000000001134

12. Domingos Dias Cicarelli.European Journal Of Anaesthesiology. El flumazenil acelera la recuperación de la anestesia con sevoflurano / remifentanilo cuando se administra a pacientes sanos no premeditadas. Noviembre 2010. Volume 27 - issue 11 - p 955-959. doi:10.1097/ eja.0b013e3283398ef9 pharmacology. https://doi.org/10.1097/ EJA.0b013e3283398ef9

13. Schwieger IM, Szlam F And Hug CC Jr. Absence of agonistic or antagonistic effect of flumazenil (ro 15-1788) in dogs anesthetized with enflurane, isoflurane, or fentanyl-enflurane. Anesthesiology 1989; 70: 477-480. https:// doi.org/10.1097/00000542-

\section{0-00018}

14. Kim YJ, Lee $\mathrm{H}$, Kim CH, Lee GY, Baik HJ and Han Jl. Effect of flumazenil on recovery from anesthesia and the bispectral index after sevoflurane/ fentanyl general anesthesia in unpremedicated patients. Korean J Anesthesiol 2012; 62: 19-23. https://doi.org/10.4097/ kjae.2012.62.1.19

15. Karakosta A, Andreotti B, Chapsa C, Pouliou a and Anastasiou E. Flumazenil expedites recovery from sevoflurane/ remifentanil anaesthesia when administered to healthy unpremedicated patients. Eur J anaesthesiol 2010; 27: 955959. https://doi.org/10.1097/ EJA.0b013e3283398ef9

16. Hiromi araki, yoshihiro fujiwara, and yasuhiro shimada. Effect of flumazenil on recovery from sevoflurane anesthesia in children premedicated with oral midazolam before undergoing herniorrhaphy with or without caudal analgesia. Journal of anestesia. Año 2005. J anesth (2005) 19:204-207. https://doi. org/10.1007/s00540-005-03144 